\title{
Evaluation of Joint Fires Management Process in Long Range Fires
}

\author{
$\underline{\text { T. A. Au }}^{\mathrm{a}}$ and E. H. S. Lo ${ }^{\mathrm{a}}$ \\ ${ }^{a}$ Joint and Operations Analysis Division, Defence Science and Technology Group, Department of Defence, \\ Australia \\ Email: andrew.au@dst.defence.gov.au
}

\begin{abstract}
Future battlespace is likely to be congested with all types of ordnance and platforms (friendly, neutral and enemy) to perform missions of simultaneous fires. Depending on the scale and complexity of the operation, both airspace deconfliction and ground clearance may require coordination of multiple air and land entities. Australian Defence Force's (ADF's) potential acquisition of a land-based long range fires (LRF) capability will present numerous battlespace challenges across an enlarged and congested battlespace. While capable of operating at long ranges (engaging targets up to $300 \mathrm{~km}$ away) and high altitudes (far in excess of routinely established levels for coordination altitude), the targeting sequence for striking time sensitive targets using LRF is complicated by coordination and deconfliction requirements with friendly forces.
\end{abstract}

While acknowledging the deconfliction challenges, an opportunity existed to examine ADF's current joint warfighting paradigm and assess two operating concepts for airspace management to facilitate long range strike. Targeting efficiency is contingent on streamlined interactions between key entities to facilitate effective information exchanges. For each concept, three target locations within an illustrative scenario were considered for coordination and deconfliction of fires. The sequence of activities, decisions and associated communication events were recorded. Social network analysis was applied to investigate workflows and evaluate key entities and patterns of communication for battlespace management.

Compared to Condition 1 (airspace managed by the Air and Space Operations Centre), Condition 2 (airspace managed by the Joint Task Force) was found to improve the interaction by reducing numbers of steps and reliance on fewer key entities. For all three cases of target location, the quality of activity execution was improved in Condition 2 by minimising the overlap in responsibilities, thus alleviating the coordination requirements. Decision delegation in Condition 2 suggested improved responsiveness in airspace management, but also revealed the need to rebalance the skills of airspace management.

Despite initial results indicating improved workflows in Condition 2, this assessment is by no means conclusive but provides the groundwork for further validation and refinement of airspace control. Future analysis will allow evaluation of new and future operational concepts, and test various what-if scenarios to improve operational effectiveness and efficiency of airspace usage in a congested and contested airspace.

Keywords: Joint fires, long range fires, airspace management, battlespace deconfliction, social network analysis 


\section{INTRODUCTION}

Currently, the Australian Defence Force (ADF) is considering procurement of a land-based long range fires (LRF) capability (Commonwealth of Australia, 2016). While LRF will equip Army with its own long range strike capability, the acquisition presents numerous challenges as to how joint fires are managed in future operational environments. LRF operates at long ranges (engaging targets up to $300 \mathrm{~km}$ away) and high altitudes (far in excess of routinely established levels for coordination altitude (CA)), thus challenging current operational concepts based on mortars and artillery use. In these concepts, Air Force has traditionally leveraged its advantages in the deep with Army operating in the close battle (Laughbaum, 1999).

Particularly poignant for Air Force is air operation in airspace operated by friendly sorties and the need to ensure their deconfliction. For Army, consideration may exist for clearing the ground around a more distant target. Both airspace deconfliction and ground clearance may require coordination of multiple Air Force and Army entities depending on the scale and complexity of the operation. The management process of LRF is further complicated if employed against time sensitive targets (TSTs) (Fyfe, 2005). These high priority targets warrant rapid response due either to the threat posed to friendly forces or their high strategic or operational importance. As a result, the management options and solutions need to be developed in much shorter time compared to planned (deliberate) targets.

While acknowledging the deconfliction challenges, an opportunity existed to examine the current ADF's joint warfighting paradigm and explore alternative arrangements for airspace management to facilitate long range strike (Lo et al., 2017). This paper reports on the analytical findings associated with LRF management processes in future joint operational environments. Two operating concepts, differing in how the airspace is managed, are discussed. For each concept, three target locations within the battlespace are considered for coordination and deconfliction of fires.

\section{DATA COLLECTION}

A workshop involving subject matter experts (SMEs) was held to elicit activity sequences between key entities to enable LRF strike for six representative configurations. The capture of information exchange requirements (IERs) enabled the application of social network analysis (SNA) to compare two alternative arrangements for airspace management.

\subsection{Two Conditions against Three Instances of Target Locations}

Airspace use above CA is traditionally dominated by fixed wing assets and hence managed by the Air and Space Operations Centre (AOC) (Royal Australian Air Force, 2006). Increased airspace use by Army and Navy will necessitate better integration between services to ensure its efficient use in achieving campaign objectives. An illustrative, high-level scenario was developed to provide context for facilitated discussions. Given broad LRF related concepts, two conditions were considered:

1. Condition 1: airspace above CA managed by the AOC; and

2. Condition 2: airspace above CA managed by the Joint Task Force (JTF).

For each condition, three instances of target location were considered as illustrated in Figure 1 where the target is positioned in:

1. Coalition (C) Brigade (BDE) area of operations (AO);

2. Australian (AS) BDE AO; and

3. JTF AO (outside of both C BDE and AS BDE AOs).

For the two conditions and three cases, the LRF weapon remained located in $\mathrm{C} B \mathrm{BDE} \mathrm{AO}$. The combination of two conditions and three target locations resulted in consideration of six overall configurations.

\subsection{The Method}

The collection method involved an interactive facilitated workshop with SMEs identifying operating nodes (entities) and IERs as follows:

1. LRF entities: sensor, C BDE Joint Fires and Effects Coordination Centre (JFECC), AS BDE JFECC, JTF

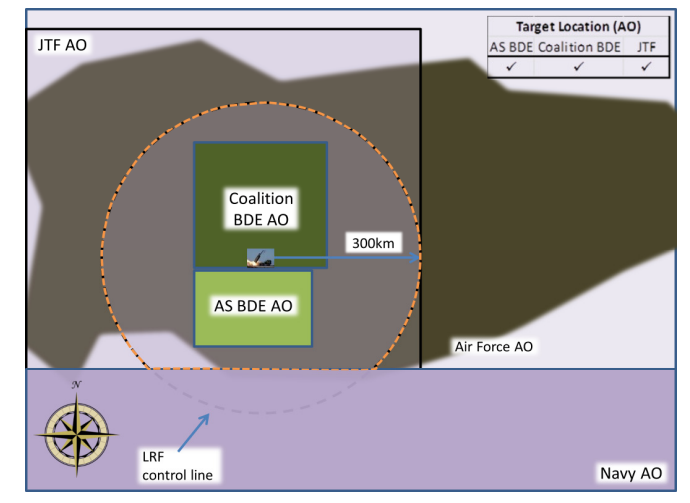

Figure 1. LRF weapon and target locations 
JFECC, Air Support Operations Centre (ASOC), LRF, AOC, and Control and Reporting Centre (CRC).

2. LRF IERs: Target (TGT) set, TGT identification (ID), data input, request for fire, mission data, request clear ground (CL-G), CL-G authorisation (Auth), request clear air (CL-A), CL-A Auth, report of ready, decision to fire, report of shot, engage, battle damage assessment (BDA), confirm end of mission (EOM), and EOM report.

Analytical models were constructed in sequence diagrams, highlighting associations between individual entities and IERs. The representation of evolving interactions enabled estimation of communications frequency between entities in the LRF workflows. While security classifications of the sequence diagrams restrict further description, this paper was intentionally limited to discussions of the statistical measures at the unclassified level.

\section{SOCIAL NETWORK ANALYSIS}

SNA is useful for studying the behaviour of military command and control (C2) networks through a representation based on nodes and edges (Batagelj \& Mrvar, 2003). Individuals or entities are represented as nodes while edges characterise relationships. This approach can be applied to assess important properties of an organisational structure in the design to improve overall work practice efficiency.

The difference between the two conditions of airspace management modified the set of key entities. In Condition 2, the AOC devolved the airspace control responsibility above CA to the ASOC. In terms of communications volume, Figures 2, 4 and 6 suggest C BDE JFECC and JTF JFECC are important nodes under Condition 1 and thus potentially critical to LRF system operation. C BDE JFECC in Figures 3, 5 and 7 appears to be a critical network hub under Condition 2 .

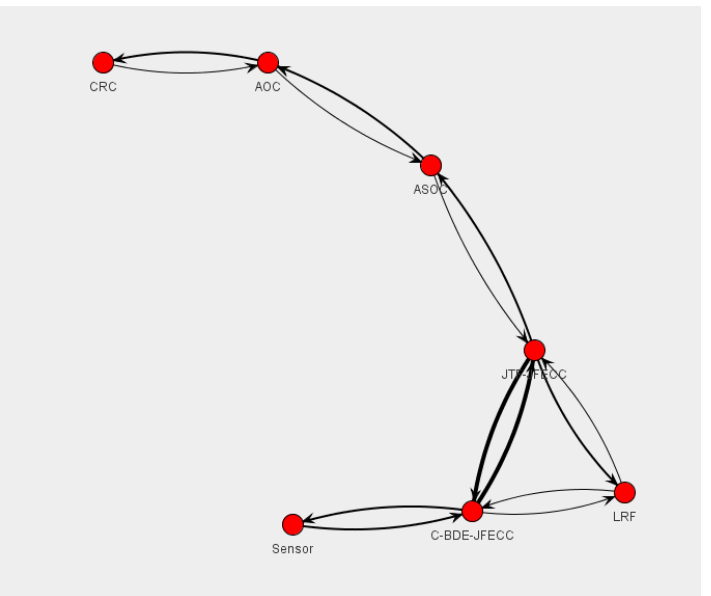

Figure 2. Cond 1 Case 1, target in $\mathrm{C} B \mathrm{BDE} \mathrm{AO}$

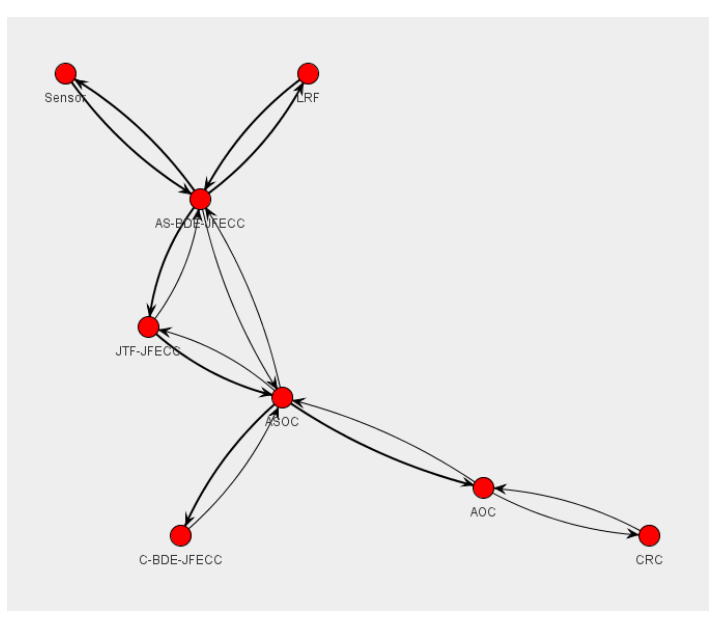

Figure 4. Cond 1 Case 2, target in AS BDE AO

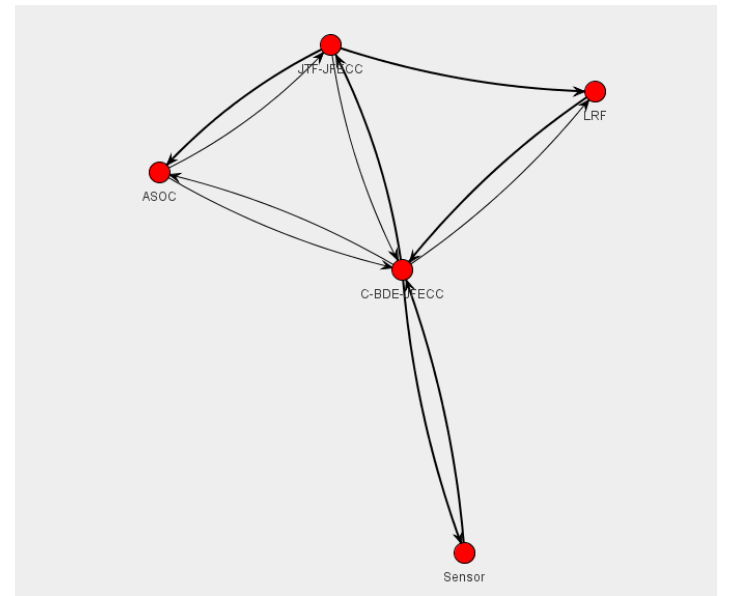

Figure 3. Cond 2 Case 1, target in $\mathrm{C}$ BDE AO

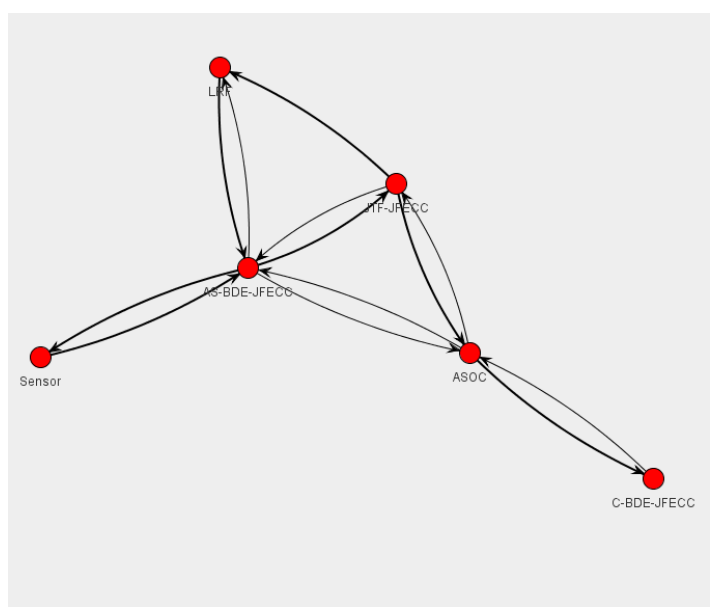

Figure 5. Cond 2 Case 2, target in AS BDE AO 


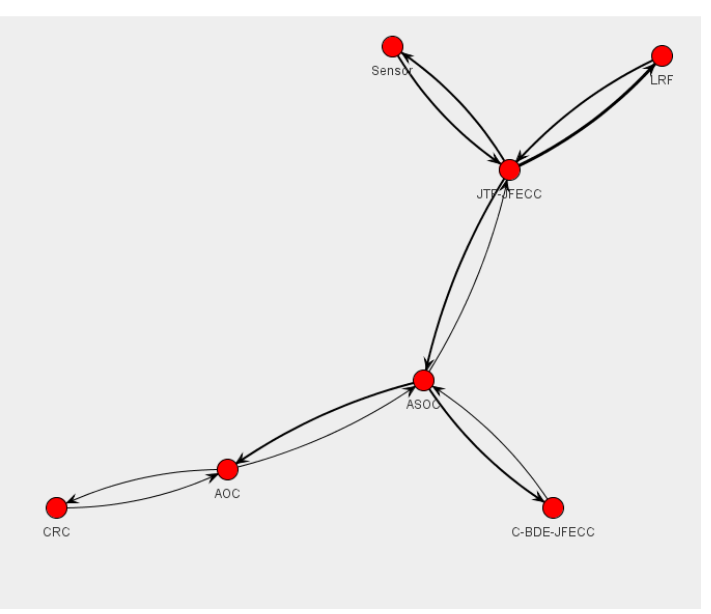

Figure 6. Cond 1 Case 3, target in JTF AO

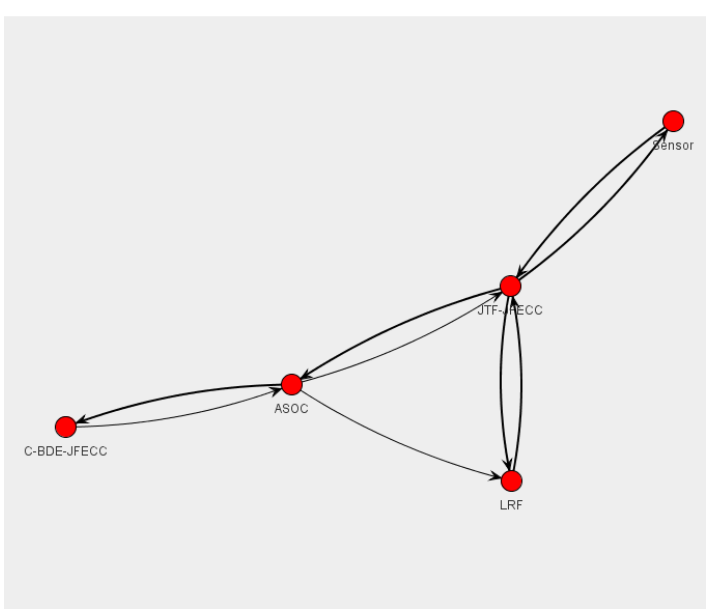

Figure 7. Cond 2 Case 3, target in JTF AO

\subsection{From CL-A Request to Authorisation}

Condition 2 changed the procedures for obtaining CL-A authorisation and the set of key entities for LRF strike. Table 1 compares the numbers of steps and associated entities for all six situations.

Table 1. Numbers of steps and key entities from CL-A request to authorisation

\begin{tabular}{|c|c|c|c|c|c|c|c|}
\hline & \multicolumn{2}{|c|}{ Case 1 } & \multicolumn{3}{c|}{ Case 2 } & \multicolumn{2}{c|}{ Case 3 } \\
\hline Condition 1 & No. of steps & No. of entities & No. of steps & No. of entities & No. of steps & No. of entities \\
\hline Condition 2 & 12 & 5 & 13 & 6 & 10 & 5 \\
\hline
\end{tabular}

These figures suggest that Condition 2 is an elegant solution for step reduction with fewer IERs and entities involved in the CL-A procedures in all three cases of target location. Information exchanges with external entities can be time-consuming, especially when warfighters are concurrently dealing with other critical activities. Each step adds potential delay or increases the risk of injected error. Reducing the number of steps may decrease the total time for CL-A authorisation and diminish the possibility of error injection. However, a disadvantage of a smaller number of steps related to this request might be the loss of real-time interaction with the AOC for essential airspace information in the vicinity of the LRF strike.

\subsection{Operational Loading}

A comparison can be made on entity loadings across airspace management conditions for each case of target location (Au \& Lo, 2009). The operational loading tables in Tables 2 to 4 count operations performed by the associated entities. A summary of operations gives an insight into possible sources of variation in workload and indication of likely stress points in terms of demand. A common trait observed in all three cases is the high operational loading in the JFECC headquarters managing the AO with the resident target, i.e. $\mathrm{C}$ BDE JFECC for a target in the C BDE AO.

Table 2. Operational loading for Case 1 - Same AO (LRF strikes a target in C BDE AO)

\begin{tabular}{|c|c|c|c|c|c|c|c|c|c|c|c|c|}
\hline \multirow{2}{*}{$\begin{array}{c}\text { Loading } \\
\text { Condition }\end{array}$} & \multicolumn{2}{|c|}{ Operations } & \multicolumn{2}{|c|}{ Transmit } & \multicolumn{2}{|c|}{ Receive } & \multicolumn{2}{|c|}{ Decision } & \multicolumn{2}{|c|}{ Request } & \multicolumn{2}{|c|}{ Total } \\
\hline & 1 & 2 & 1 & 2 & 1 & 2 & 1 & 2 & 1 & 2 & 1 & 2 \\
\hline Sensor & 2 & 2 & 2 & 2 & 2 & 2 & - & - & - & - & 6 & 6 \\
\hline C BDE JFECC & 1 & 1 & 7 & 6 & 7 & 6 & 3 & 4 & 2 & 1 & 20 & 18 \\
\hline JTF JFECC & - & - & 8 & 5 & 6 & 3 & 1 & - & 2 & 1 & 17 & 9 \\
\hline ASOC & - & - & 3 & 2 & 3 & 3 & - & - & 1 & 1 & 7 & 6 \\
\hline LRF & 1 & 1 & 2 & 2 & 3 & 3 & - & - & - & - & 6 & 6 \\
\hline AOC & - & - & 3 & - & 3 & - & - & - & 1 & - & 7 & 0 \\
\hline CRC & - & - & 1 & - & 2 & - & 1 & - & - & - & 4 & 0 \\
\hline
\end{tabular}


Apart from identifying $\mathrm{C}$ BDE JFECC as having the highest total loading for Case 1, Table 2 also records a significant level of communications activity by the JTF JFECC due to its operational control of the LRF capability, a weapon force assigned to C BDE headquarters (Commonwealth of Australia, 2009). Condition 2 did not impose additional operational loading on the ASOC while the AOC was disengaged from CL-A authorisation. Both the C BDE and JTF JFECCs have reduced operational loading in Condition 2.

Table 3. Operational loading for Case 2 - Different AO (LRF strikes a target in AS BDE AO)

\begin{tabular}{|c|c|c|c|c|c|c|c|c|c|c|c|c|}
\hline \multirow{2}{*}{$\begin{array}{c}\text { Loading } \\
\text { Condition }\end{array}$} & \multicolumn{2}{|c|}{ Operations } & \multicolumn{2}{|c|}{ Transmit } & \multicolumn{2}{|c|}{ Receive } & \multicolumn{2}{|c|}{ Decision } & \multicolumn{2}{|c|}{ Request } & \multicolumn{2}{|c|}{ Total } \\
\hline & 1 & 2 & 1 & 2 & 1 & 2 & 1 & 2 & 1 & 2 & 1 & 2 \\
\hline Sensor & 2 & 2 & 2 & 2 & 2 & 2 & - & - & - & - & 6 & 6 \\
\hline C BDE JFECC & - & - & 1 & 1 & 2 & 2 & 1 & 1 & - & - & 4 & 4 \\
\hline JTF JFECC & - & - & 5 & 5 & 3 & 3 & - & - & 1 & 1 & 9 & 9 \\
\hline ASOC & - & - & 6 & 4 & 5 & 4 & - & 1 & 3 & 2 & 14 & 11 \\
\hline LRF & 1 & 1 & 2 & 2 & 3 & 3 & - & - & - & - & 6 & 6 \\
\hline AOC & - & - & 3 & - & 3 & - & - & - & 1 & - & 7 & 0 \\
\hline CRC & - & - & 1 & - & 2 & - & 1 & - & - & - & 4 & 0 \\
\hline AS BDE JFECC & 1 & 1 & 6 & 6 & 6 & 6 & 4 & 4 & 1 & 1 & 18 & 18 \\
\hline
\end{tabular}

For Case 2, AS BDE JFECC is identified in Table 3 as having the highest level of activity followed by the ASOC due especially to communications requirements in processing the request. As explained above, the AOC recorded no involvement in CL-A authorisation in Condition 2 so no operational loading is incurred. In Condition 2, the ASOC is given decision authority but records less operational loading.

Table 4. Operational loading for Case 3 - JTF AO (LRF strikes a target in JTF AO)

\begin{tabular}{|c|c|c|c|c|c|c|c|c|c|c|c|c|}
\hline \multirow{2}{*}{$\begin{array}{c}\text { Loading } \\
\text { Condition }\end{array}$} & \multicolumn{2}{|c|}{ Operations } & \multicolumn{2}{|c|}{ Transmit } & \multicolumn{2}{|c|}{ Receive } & \multicolumn{2}{|c|}{ Decision } & \multicolumn{2}{|c|}{ Request } & \multicolumn{2}{|c|}{ Total } \\
\hline & 1 & 2 & 1 & 2 & 1 & 2 & 1 & 2 & 1 & 2 & 1 & 2 \\
\hline Sensor & 2 & 2 & 2 & 2 & 2 & 2 & - & - & - & - & 6 & 6 \\
\hline C BDE JFECC & - & - & 1 & 1 & 2 & 2 & 1 & 1 & - & - & 4 & 4 \\
\hline JTF JFECC & 1 & 1 & 7 & 6 & 5 & 5 & 3 & 3 & 1 & 1 & 17 & 16 \\
\hline ASOC & - & - & 5 & 4 & 4 & 3 & - & 1 & 2 & 1 & 11 & 9 \\
\hline LRF & 1 & 1 & 2 & 2 & 3 & 3 & - & - & - & - & 6 & 6 \\
\hline AOC & - & - & 2 & - & 3 & - & - & - & 1 & - & 6 & 0 \\
\hline CRC & - & - & 1 & - & 1 & - & 1 & - & - & - & 3 & 0 \\
\hline
\end{tabular}

In Case 3, JTF JFECC is shown in Table 4 to incur the highest level of activity followed by the ASOC. With the AOC disengaged in Condition 2, the ASOC has the authority to make CL-A decision while reducing workload for communications.

\subsection{Social Interactions and Measures}

In order to explore the relative importance of these entities (or nodes) to the social network, we characterise the LRF workflows using two social network metrics - betweenness centrality and sociometric status (Lo et al., 2011). Centrality is an overall indication of how close a node is in terms of geodesic distance from other nodes, i.e., the length of the shortest path connecting the two nodes. A well-connected node will reveal its social importance in the network (Tapiero \& Lewin, 1973). Sociometric status is a measure of node activity indicating a given node's contribution to the overall amount of communication in the network. In developmental psychology, sociometric status reflects one's popularity or rejection by peers.

Table 5 shows the results for betweenness centrality measures for the three cases of target location against the two conditions of airspace management for LRF strike. In each configuration, the centrality value of the mean plus one standard deviation is used to define 'key' entities in the given network (Houghton et al., 2006). Lower centrality scores are observed in Condition 2 than in Condition 1. 
Table 5. Betweenness centrality measures for long range fires

\begin{tabular}{|c|c|c|c|c|c|c|}
\hline Case & \multicolumn{2}{|c|}{$\mathbf{1}$} & \multicolumn{2}{c|}{$\mathbf{2}$} & $\mathbf{3}$ \\
\hline Condition & $\mathbf{1}$ & $\mathbf{2}$ & $\mathbf{1}$ & $\mathbf{2}$ & $\mathbf{1}$ & $\mathbf{2}$ \\
\hline Sensor & 0 & 0 & 0 & 0 & 0 & 0 \\
\hline C BDE JFECC & 10 & 8.5 & 0 & 0 & 0 & 0 \\
\hline JTF JFECC & 18 & 0.5 & 0 & 1 & 18 & 8 \\
\hline ASOC & 16 & 0 & 28 & 8 & 22 & 6 \\
\hline LRF & 0 & 0 & 0 & 0 & 0 & 0 \\
\hline AOC & 10 & - & 12 & - & 10 & - \\
\hline CRC & 0 & - & 0 & - & 0 & - \\
\hline AS BDE JFECC & - & - & 22 & 12 & - & - \\
\hline
\end{tabular}

Table 6 records the scores for sociometric status measures against the three cases of target location and two conditions of airspace management. The 'key' entities highlighted in each configuration identify those with sociometric status exceeding the mean plus one standard deviation. Distributions of these scores indicate that the $\mathrm{C} 2$ node responsible for the AO containing the target dominates the activity of CL-A authorisation. Although taking the decision authority in Condition 2, the ASOC is not as influential as in Condition 1.

Table 6. Sociometric status measures for long range fires

\begin{tabular}{|c|c|c|c|c|c|c|}
\hline Case & \multicolumn{2}{|c|}{$\mathbf{1}$} & \multicolumn{2}{c|}{$\mathbf{2}$} & \multicolumn{2}{c|}{$\mathbf{3}$} \\
\hline Condition & $\mathbf{1}$ & $\mathbf{2}$ & $\mathbf{1}$ & $\mathbf{2}$ & $\mathbf{1}$ & $\mathbf{2}$ \\
\hline Sensor & 0.667 & 1 & 0.571 & 0.8 & 0.667 & 1 \\
\hline C BDE JFECC & 2.333 & 3 & 0.429 & 0.6 & 0.5 & 0.75 \\
\hline JTF JFECC & 2.333 & 2 & 0.857 & 1.6 & 2 & 2.75 \\
\hline ASOC & 1 & 1.25 & 1.571 & 1.6 & 1.5 & 1.75 \\
\hline LRF & 0.833 & 1.25 & 0.571 & 1 & 0.833 & 1.25 \\
\hline AOC & 1 & - & 0.714 & - & 0.833 & - \\
\hline CRC & 0.5 & - & 0.286 & - & 0.333 & - \\
\hline AS BDE JFECC & - & - & 1.857 & 2.4 & - & - \\
\hline
\end{tabular}

\section{CONCLUDING REMARKS}

The future battlespace is likely to be congested with all types of ordnance and platforms to perform missions of simultaneous fires. LRF operating at long ranges and high altitudes will add to the complexity of traditional airspace management concepts. The basic targeting sequence will particularly challenge prosecution of TSTs due to requirements to ensure proper coordination and deconfliction with friendly forces while working under constrained times.

The efficiency of the targeting process is contingent on streamlined interactions of key entities to facilitate effective information exchanges. An exploratory experiment was conducted to collect data from SMEs focusing on battlespace management for LRF. The sequence of activities, decisions and associated communication events were elicited as the scenario unfolded. This paper applied SNA to investigate LRF workflows for two conditions against three instances of target location. Examination of interactions herein revealed key entities and patterns of communication in each condition of airspace management.

It was found that Condition 2 of airspace management improved the interaction with CL-A requestor by reducing the number of steps involved with external entities. These are often redundant communications activities that can be eliminated to decrease the total time for CL-A authorisation. Condition 2 involves fewer key entities, leading to less split responsibilities in airspace management from the AOC and ASOC. Minimising the overlap in responsibilities can reduce the complexity of coordination requirements and improve the quality of activity execution (Dumas et al., 2013). This finding is justified by the observation 
that Condition 2 did not impose additional operational loading on key entities for all three cases of target location.

In Condition 2, airspace management decisions are delegated to the ASOC to improve responsiveness in activity execution, making it less susceptible to error. It means that some airspace specialists must be included in the ASOC staff to deepen the skills of airspace management. This expertise is required to increase the processing speed and accuracy of CL-A routines, instead of relying on the remote AOC. The detachment of the AOC from the LRF workflows in Condition 2 also suggests reduced resources required in the AOC for processing CL-A authorisation. Condition 2 indicates that empowering the ASOC staff to take airspace management decisions may result in streamlined operations with reduced processing times.

This assessment however is not conclusive despite initial results indicating improved LRF workflows in Condition 2. The information exchanges were elicited at a relatively high level of abstraction and are considered insufficient to reveal the complexity of airspace management procedures. An example is the loss of direct interaction with the AOC in Condition 2. An alternative mechanism should be in place to facilitate situational awareness of the ASOC. The ability to access real-time essential airspace information is a prerequisite for decision making in airspace management especially in the vicinity of an LRF strike. For further model refinements, developing an executable model based on these findings can capture and broaden the understanding of LRF system in hypothetical battlespace. Studies of this form can inform on the additional workload and skillset requirements on the augmented ASOC and the additional demands on fundamental inputs to capability. Execution of such a model would enable evaluation of new and future operational concepts, and test various what-if scenarios. This future effort will attempt to improve operational effectiveness and efficiency of airspace usage in a congested and contested airspace.

\section{ACKNOWLEDGEMENTS}

The authors would like to thank Mr Vikram Koduru for generating the results for social network measures using the Social Network Analysis of Command and Control (SNAC2) tool.

\section{REFERENCES}

Au, T.A., and Lo, E.H.S. (2009). Evaluation of Command and Control Activity for Air Operations. Proceedings of Human Factors and Ergonomics Society of Australia Conference, Melbourne, Australia.

Batagelj, V. and Mrvar, A. (2003). Pajek - Analysis and Visualisation of Large Networks . Graph Drawing Software, M. Jünger and P. Mutzel (Eds.), Berlin: Springer.

Commonwealth of Australia (2009). ADDP 00.1 Command and Control. Defence Publishing Service, Department of Defence.

Commonwealth of Australia (2016). 2016 Defence White Paper. Department of Defence, Australian Government.

Dumas, M., La Rosa, M., Mendling, J., and Reijers, H.A. (2013). Fundamentals of Business Process Management. Springer-Verlag Berlin Heidelberg..

Fyfe, J.M. (2005). The Evolution of Time Sensitive Targeting: Operation Iraqi Freedom Results and Lessons. Research Paper 2005-02, Airpower Research Institute, College of Aerospace Doctrine, Research and Education, Air University.

Houghton, R.J., Baber, C., McMaster, R., Stanton, N.A., Salmon, P., Stewart, R., and Walker, G. (2006). Command and Control in Emergency Services Operations: A Social Network Analysis. Ergonomics, 49 (12-13), Taylor \& Francis.

Laughbaum, R.K. (1999). Synchronising Airpower and Firepower in the Deep Battle. CADRE Paper, College of Aerospace Doctrine, Research, and Education, Air University, Air University Press.

Lo, E.H.S., Au, T.A., Hoek, P.J., and Eberl, L. (2011) Analysis of Evolving Team Interactions in Dynamic Targeting. International Journal of Intelligent Defence Support Systems, 4(4), 309-327.

Lo, E.H.S., Janczura, C., Au, T.A., and Filippidis, D. (2017). Battlespace Management for Long Range Fires: Table Top Experiment (TTX) 1/17 Findings. DSTG Technical Note, Defence Science and Technology Group (submitted for approval).

Royal Australian Air Force (2006). AAP 1002 The Operational Air Doctrine Manual. Headquarters Air Command, Glenbrook, NSW.

Tapiero, C.S. and Lewin, A.Y. (1973). The Concept and Measurement of Centrality - An Information Approach. Decision Sciences, 4(3), July, 314-328. 